\title{
Parametric Resonance of Entropy Perturbations in Massless Preheating
}

\author{
Hossein Bazrafshan Moghaddam, Robert H. Brandenberger, Yi-Fu Cai, and Elisa G.M. Ferreira ${ }^{1, \text { ศ }}$ \\ ${ }^{1}$ Department of Physics, McGill University, Montreal, QC H3A 2T8 Canada
}

\begin{abstract}
Here, we revisit the question of possible preheating of entropy modes in a two field model with a massless inflaton coupled to a matter scalar field. Using a perturbative approximation to the covariant method we demonstrate that there is indeed a parametric instability of the entropy mode which then at second order leads to exponential growth of the curvature fluctuation on superHubble scale. Back-reaction effects shut off the induced curvature fluctuations, but possibly not early enough to prevent phenomenological problems. This confirms previous results obtained using different methods and resolves a controversy in the literature.
\end{abstract}

\section{INTRODUCTION}

The "reheating" phase is an important period of inflationary cosmology. It describes the transfer of the energy density between the inflaton field, the field which drives the inflationary expansion of space, and regular matter. Without reheating, inflation would leave behind a universe devoid of matter, not the kind of universe we observe. The energy transfer between the inflaton field and regular matter is a consequence of couplings between the inflaton field $\phi$ and "regular matter". For simplicity, in studies of reheating the regular matter which consists of fermions, gauge fields and scalars is usually modelled by a scalar field $\chi$ which has some direct couplings with $\phi$. The energy transfer at the end of the period of exponential expansion of space from the inflaton field $\phi$ to regular matter $\chi$ was initially studied using first order perturbation theory [1]3. However, such an analysis does not take into account the fact that the inflaton field $\phi$ at the end of the period of inflation is in a highly excited homogeneous condensate state and is not an assembly of free perturbative quanta. A treatment of the energy transfer which takes into account the coherence of the inflaton condensate was proposed in 4,5 . In these works, it was realized that the energy transfer from $\phi$ and $\chi$ is driven by a parametric resonance instability. This instability was worked out in detail in 6 [ ing" was coined to describe this energy transfer process. The instability is exponential and typically leads to an energy transfer which is rapid on the Hubble time scale $H^{-1}$, where $H$ is the Hubble expansion rate at the end of the period of inflation. On the other hand, the state of $\chi$ after preheating is not thermal, and to achieve a thermal state of matter particles a second stage of reheating, namely the thermalization stage is required. For recent reviews of reheating see e.g. 9, 10. We will not discuss the thermalization stage further since we are interested in this paper in certain effects of the preheating phase.

From the mathematical point of view, the key aspect of preheating is that the oscillations in time of the inflaton field $\phi$ after the end of inflation lead to a periodically varying contribution to the mass term of the $\chi$ field. The equation of motion for $\chi$ thus falls into the category of those described by Floquet theory [11, which states that there are bands of Fourier modes of $\chi$ which experience exponential growth. Since the inflaton field $\phi$ also couples to gravity, the oscillations of $\phi$ lead to a periodically varying contribution to the mass term in the equation of motion for cosmological perturbations, as was first pointed out in [12] and [13]. Hence, there is the possibility that preheating can lead to a parametric instability in the amplitude of cosmological perturbations, even on scales which are super-Hubble at the end of inflation. If this were true, it would completely change the usual predictions of inflationary models. In fact, the presence of a resonant instability of cosmological fluctuation modes could lead to an amplitude of fluctuations which is much larger than the observed value, thus placing constraints on inflationary models.

In 14] (see also [15 17]), it was shown that in models with only adiabatic fluctuations there is no instability of curvature fluctuations on super-Hubble scales. This is related to the conservation of the comoving curvature fluctuation variable $\zeta$ on super-Hubble scales [18, 19] (see also 20 22 for early work). However, in certain two field models of inflation it was argued in [23 26] that in the case of "massless preheating" (the inflaton having vanishing mass) there will be a preheating instability for the entropy fluctuation mode, even on super-Hubble scales [61. In fact, it was shown in [28, 29] that back reaction effects will not be strong enough to shut off the instability before the entropy mode has become dominant. The presence of a preheating instability for the entropy mode was confirmed in the analyses of [30] and [31, extended to the case of multifield generalized Einstein models in [32], and applied to certain examples in 33].

However, there remain concerns about the conclusions of 14. An analysis using the "separate universe" method [34 argues that the preheating of entropy fluctuations is less effective. An analysis using the $\delta N$ formalism, a method which is closely related to the separate universe approach, finds that there is parametric resonance of the entropy mode [35, although in a subsequent paper 36] the same authors do not find substantial effects on the curvature fluctuations. In addition, little amplification of the curvature fluctuations is observed in the numerical 
work of 37] which was based on a numerical implementation of the $\delta N$ formalism.

Since the entropy mode sees a growing curvature fluctuation on super-Hubble scales, any parametric resonance instability of an entropy mode can lead to an exponential growth of the curvature fluctuation during the preheating stage. This is a potentially disastrous effect since the fluctuations could well grow to become larger than the observed values. Hence, from the point of view of inflation model building it is very important to determine whether the parametric resonance instability of the entropy mode is robust. The goal of this paper is to reconsider this question using different methods than have been used before. Specifically we will make use of the "covariant formalism", a formalism developed in 38 (see also 39] for earlier related work), a formalism which can be applied even non-perturbatively (we, however, will use a perturbative truncation of the formalism).

Our study shows that the preheating of entropy modes is indeed effective in the massless preheating toy model which we consider, and that this leads to an exponentially growing contribution to the curvature fluctuation. Since we have shown that our equations are the perturbative limit of a consistent non-perturbative formalism, we now have a better reason for arguing that the instability we find will extend beyond the perturbative treatment.

Our goal is to demonstrate an instability of the model on cosmological scales. Our methods obviously break down once the nonlinear regime is reached. At this point, numerical methods used to study nonlinear preheating effects (see e.g. 40 for the first numerical code for studying preheating which includes the metric fluctuations, and [37, 41, 46]) would have to be applied. These nonlinear effects, however, cannot reduce the amplitude of curvature perturbations on cosmological scales, and hence we do not consider them.

We emphasize that our results are not new. They confirm the conclusions reached in earlier work (specifically in [14]). However, our paper resolves a controversy in the literature about the conclusions which were reached in the earlier works.

\section{MASSLESS PREHEATING}

In [14 necessary conditions for the effectiveness of preheating of the entropy mode of metric fluctuations have been discussed. One of the conditions is that there is efficient parametric resonance in the matter sector in the absence of gravitational fluctuations. A model of massless inflation satisfies this condition. Hence, in this paper we consider a toy model containing a massless inflaton field $\phi$ coupled to a massive matter field $\chi$ with a potential

$$
V(\phi, \chi)=\frac{\lambda}{4} \phi^{4}+\frac{1}{2} g^{2} \phi^{2} \chi^{2} .
$$

The interaction term $\phi^{2} \chi^{2}$ allows for the decay of the coherent inflaton configuration $\phi$ into massive $\chi$ excitations.

Up to the mass term for $\chi$ (which we will neglect in the following) our model is conformally invariant. Thus, via a conformal transformation we can map our model into one living in Minkowski space-time, and then study preheating in Minkowski space-time. This is technically much simpler than performing the calculation in the original variables in an expanding universe. As pointed out first by [47, the structure of resonance in the matter sector depends in a crucial way on the relation between the coupling constants $\lambda$ and $g^{2}$. Indeed the only parameter responsible for the structure of resonance is the ratio $\frac{g^{2}}{\lambda}$.

We are interested in studying the evolution of the entropy perturbation in this two field model. To do this we first need to study the evolution of the quantum field $\chi$ in the background of the classical field $\phi$. This will be done in the rest of this section.

We consider the case in which the zero mode of the $\chi$ field is zero and therefore $\chi$ has no effect on the inflationary dynamics in the absence of quantum fluctuations. However, quantum fluctuation of this field are continuously excited. Since the equation of motion for $\chi$ is linear in $\chi$ each Fourier mode evolves separately. We are interested in modes whose wavelength today corresponds to cosmological scales. The wavelength is smaller than the Hubble radius early during the inflationary phase, grows relative to the Hubble radius as a consequence of the accelerated expansion of space during the period of inflation, and exits the Hubble radius a number of e-folding times before the end of inflation. At that point, the oscillations of the quantum fluctuations freeze out and the modes can be squeezed.

Later on we will have to take into account the fact that the ensemble of large scale fluctuations of the $\chi$ field will generate an effective $\chi$ background in which smaller scale $\chi$ modes live. We will find this background by averaging over the large-scale fluctuations.

\section{Evolution of the Inflaton $\phi$}

First we review the dynamics of $\phi$ after the end of the period of inflation. During inflation the effective potential for the inflaton is $\frac{\lambda}{4} \phi^{4}$. Therefore the Klein-Gordon for the classical inflaton field $\phi$ is

$$
\ddot{\phi}+3 H \dot{\phi}+\lambda \phi^{3}=0
$$

where $H$ is the Hubble parameter and " " " is the derivative with respect to physical time. For further simplification we work with conformal time $\eta$ defined via

$$
a d \eta=d t .
$$


Then as we mentioned before we do a conformal transformation

$$
a \phi=\varphi .
$$

If we rewrite equation (2), we find

$$
\varphi^{\prime \prime}+\lambda \varphi^{3}-\frac{a^{\prime \prime}}{a} \varphi=0,
$$

where " 1 " denotes the derivative with respect to conformal time.

After the end of the period of slow-roll inflation, the background $\Phi$ field will start anharmonic oscillations about $\Phi=0$ since it lives in a confining potential. It is well known that for a quartic potential $\lambda \phi^{4}$ the time average of the time-averaged energy momentum tensor is traceless and hence the equation of state (averaged over an oscillation period) is the same as for radiation. Hence $a(\eta) \sim \eta$, and therefore the last term in the equation (3) vanishes. Thus we obtain

$$
\varphi^{\prime \prime}+\lambda \varphi^{3}=0
$$

which has periodic solutions. To find these solutions we introduce the dimensionless conformal time

$$
x \equiv \sqrt{\lambda} \tilde{\varphi} \eta,
$$

where $\tilde{\varphi}$ is the constant amplitude of the oscillations of $\varphi=\tilde{\varphi} f(x)$. The solution of equation (4) can be written in terms of Jacobi elliptic functions:

$$
\varphi=\tilde{\varphi} c n\left(x-x_{0}, \frac{1}{\sqrt{2}}\right) .
$$

We can approximate the elliptic cosine function by the leading term in its series expansion, $\cos (x)$, which is very good approximation as discussed in [47. Therefore the solution of the Klein-Gordon equation for the $\varphi$ field is

$$
\varphi=\tilde{\varphi} \cos (x) .
$$

Before moving on to next section, for further reference it is useful to find the form of scale factor in this theory [47. At the end of inflation and beginning of preheating the effective potential is $\lambda \phi^{4} / 4$ and the homogeneous value of $\chi$ field is zero. Thus the Friedman equation is

$$
H^{2}=\frac{8 \pi}{3 M_{p}^{2}}\left(\frac{1}{2} \dot{\phi}^{2}+\frac{\lambda \phi^{4}}{4}\right),
$$

where $H$ is the Hubble parameter. When averaging over several oscillations of the inflaton field while $\phi \ll M_{p}$ we then find

$$
a(x) \sim \sqrt{\frac{2 \pi}{3}} \frac{\tilde{\varphi}}{M_{p}} x .
$$

\section{Evolution of the Preheat Field $\chi$}

In this subsection we study the evolution of the linear mode functions of the $\chi$ field, and use the results to determine an effective background $\chi$ field which a fixed Fourier mode of the fluctuations will feel. As discussed e.g. in [29], this background is obtained by integrating over fluctuations of wavelength larger than the one we are considering.

At the classical level, the homogeneous value of the $\chi$ field is zero. The effective background which a mode with wavenumber $k$ will feel is generated by the quantum fluctuations of larger wavelengths which have exited the inflationary Hubble radius earlier, have been squeezed and decohered and hence become classical (see e.g. 48, 49]). To find this effective background, we must first solve the equation for the quantum fluctuations of the $\chi$ field. For simplicity we take the spatial sections to be flat.

As it is standard in the field, we use the formalism of quantum field theory in curved space-time. Since we are considering a free quantum field $\hat{\chi}$, we can expand the field in Fourier modes, and each Fourier mode in creation and annihilation operators $\hat{a}_{k}^{+}$and $\hat{a}_{k}$, respectively

$$
\begin{aligned}
\hat{\chi}(t, \vec{x})=\frac{1}{(2 \pi)^{3 / 2}} \int d^{3} k & {\left[\hat{a}_{k} \chi_{k}(t) \exp (-i k \cdot x)\right.} \\
+ & \left.\hat{a}_{k}^{+} \chi_{k}^{*}(t) \exp (i k \cdot x)\right],
\end{aligned}
$$

where the mode functions $\chi_{k}$ satisfy the following Fourier space Klein-Gordon equation

$$
\ddot{\chi}_{k}+3 H \dot{\chi}_{k}+\left(\frac{k^{2}}{a^{2}}+g^{2} \phi^{2}\right) \chi_{k}=0 .
$$

In conformal time and considering conformal transformation

$$
a \chi \equiv X
$$

as well as a conformal transformation of the $\phi$ field

$$
a \phi \equiv \varphi
$$

we find

$$
X_{k}^{\prime \prime}+\left[\frac{k^{2}}{\lambda \tilde{\varphi}^{2}}+\frac{g^{2}}{\lambda} f(x)-\frac{a^{\prime \prime}}{a}\right] X_{k}=0,
$$

where " " "denotes the derivative with respect to dimensionless conformal time, and $f(x)$ is the periodic with amplitude 1. As mentioned before, the last term in this equation vanishes during massless preheating and the equation becomes:

$$
X_{k}^{\prime \prime}+\left[\frac{k^{2}}{\lambda \tilde{\varphi}^{2}}+\frac{g^{2}}{\lambda} \cos ^{2}(x)\right] X_{k}=0,
$$


where we have also inserted the approximate form of $f(x)$. This equation has the structure of a Mathieu equation. To make it clear we rewrite equation (14) as follows:

$$
X_{k}^{\prime \prime}+\left[\left(\frac{k^{2}}{\lambda \tilde{\varphi}^{2}}+\frac{g^{2}}{2 \lambda}\right)+\frac{g^{2}}{2 \lambda} \cos (2 x)\right] X_{k}=0 .
$$

As we mentioned in the Introduction we will consider values of the coupling constant for which it is known that there is preheating in the matter sector. Hence, we consider the case $\frac{g^{2}}{\lambda} \simeq 2$ since in this case all long wavelength modes of the $\chi$ field are located in the instability region of the Mathieu equation 47. Therefore the solution for $X_{k}$ will be of the form

$$
X_{k}(x)=A_{1} \exp \left(\mu_{k} x\right) P_{1}(x, k)+A_{2} \exp \left(-\mu_{k} x\right) P_{2}(x, k),
$$

where $\mu_{k}$ is the so-called Floquet exponent [11] which in this case has a positive real component (the Lyapunov exponent), and $P_{1}$ and $P_{2}$ are periodic functions of $x$ with amplitude 1 which appear in the solution of the Mathieu equation [11. Note that the period is determined by the period of the inflaton field, and is independent of $k$.

Considering only the growing mode we need to determine the constant $A_{1}$ by fixing the initial conditions. Since preheating is preceded by a phase of inflationary expansion, the initial conditions for preheating are determined by the evolution of the field during inflation. This slow-roll inflation is given by a quasi exponential expansion of the universe, where the Hubble parameter is almost constant. During inflation, quantum $\chi$ field perturbations (as well as $\phi$ perturbations) are created from vacuum initial conditions on sub-Hubble scales. As the wavelengths of these fluctuations are amplified in this phase relative to the Hubble radius, they eventually exit the Hubble radius where they "freeze out" and may undergo squeezing.

To see whether squeezing occurs, we have to compare the magnitude of the induced mass term in (13), the term $\frac{g^{2}}{\lambda} f(x) X_{k}$, with the squeezing term $\frac{a^{\prime \prime}}{a} X_{k}$. Thus, the condition for squeezing is

$$
\frac{g^{2}}{\lambda}<\frac{a^{\prime \prime}}{a} .
$$

Re-expressing the derivative with respect to the rescaled time in terms of the regular time derivates, the condition 17 becomes (making use of $|\dot{H}| \ll H^{2}$ )

$$
H^{2}>g^{2} \Phi^{2} .
$$

Since during slow-roll it follows from (7) that $H^{2} \sim \Phi^{4}$, we see that (18) will be more easily satisfied for large values of the inflaton field. Thus, to see if we get squeezing we need to determine the range of values of $\Phi$ during slow-roll inflation. Specifically, we need to determine the value of $\Phi$ at the end of the slow-roll period.
During slow-roll, the second derivative term in 22 is neglected and the kinetic term is negligible in the Friedmann equation (7). Solving for the evolution of $\Phi$ in the slow-roll approximation and using the result to check when the kinetic contribution to $H$ ceases to be subdominant yields the result

$$
\varphi_{\text {end }}^{2} \sim 2(6 \pi G)^{-1}
$$

for the value of $\varphi$ at the end of the slow-roll period. We are interested whether there is squeezing for modes which exit the Hubble radius a number $N$ Hubble times before the end of inflation (for scales of cosmological interest we have $N \sim 50$ ). Making a Taylor expansion in the evolution of $\varphi$ about the endpoint of the slow-roll phase yields the lower bound

$$
\varphi(N)>\varphi_{\text {end }}\left(1+N \frac{3}{2}\right)
$$

for the value of $\varphi N$ Hubble expansion times before the end of inflation. Inserting this result into (18), we can see that the condition 18 is satisfied provided $N>2$. This means that the quantum fluctuations of the entropy modes will be squeezed between when they exit the Hubble radius and when $N=2$, i.e. essentially to the end of the slow-roll phase.

Since during the squeezing period $X_{k} \sim a$, the power spectrum of the entropy modes from inflation becomes scale invariant and the initial conditions for the $X$ modes at the beginning of the preheating phase are given by:

$$
A_{1}(k)=\frac{1}{\sqrt{2 k}} \frac{a\left(t_{R}\right)}{a\left(t_{H}(k)\right)}=H_{I} k^{-3 / 2},
$$

where $t_{R}$ is the time when inflation ends. We will normalize the scale factor such that $a\left(t_{R}\right)=1$. Also, $t_{H}(k)$ is the time of horizon crossing, with $a\left(t_{H(k)}\right)=k / H_{I}$ and $H_{I}$ the Hubble rate during inflation. With that, the solution for $X_{k}$ is:

$$
X_{k}(x) \simeq H_{I} k^{-3 / 2} \exp \left(\mu_{k} x\right) P_{1}(x, k) .
$$

Thus we can observe clearly that the $X_{k}$ mode function is exponentially growing, which in turn leads to an exponentially growing number density of $\chi$ particles. The value of the Floquet exponent as a function of $k$ is shown in Fig. 1 [47. In this figure, the vertical axis denotes the value of the Floquet exponent, the horizontal axis labels $k$. Note that for the infrared modes which we are interested in, the value of the Floquet exponent is about 0.2. Even though $\mu_{k}<1$, the time scale of the exponential instability is (while long compared to the oscillation time) short compared to the Hubble expansion time. Note that the analysis has so far been in the absence of gravitational fluctuations. 


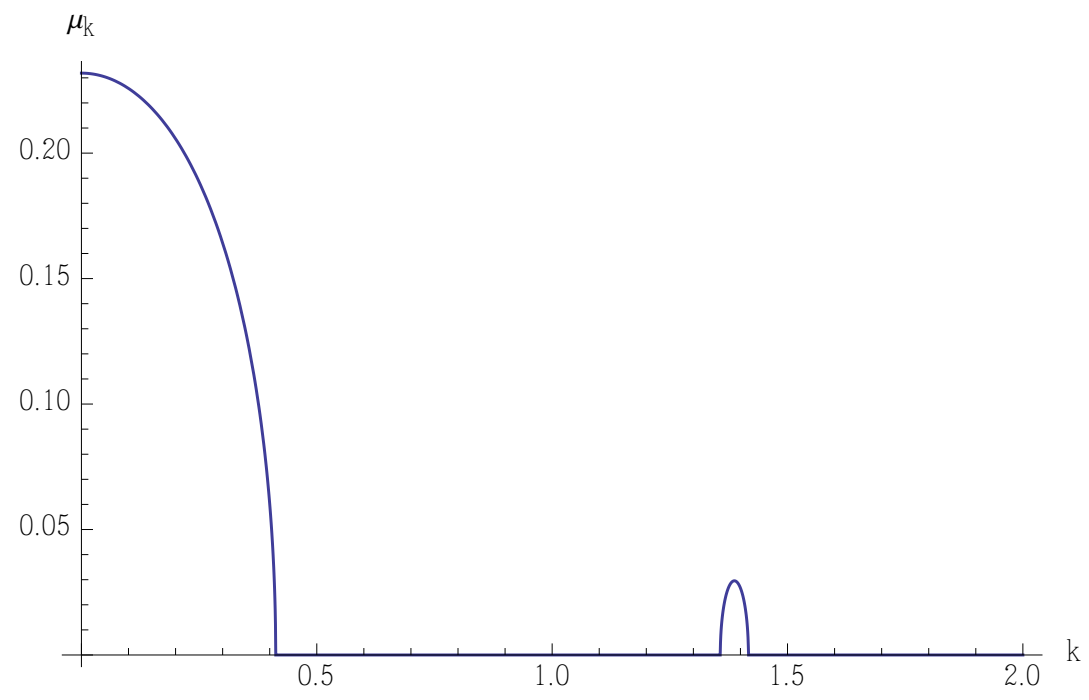

FIG. 1: Value of the Floquet exponent (vertical axis) as a function of $k$ in units of $k^{2} /\left(\lambda \tilde{\phi}^{2}\right)$.

The quantum fluctuations on large wavelength which were squeezed and classicalized after exiting the Hubble radius will form a background in which the metric fluctuations evolve. To find the effective background value which a mode with wavenumber $k$ feels, we need to integrate over all wavelengths larger than $k^{-1}$ [62 Thus, we define the effective background of the $\chi$ field on a scale $k$ as

$$
\chi_{\mathrm{eff}}(k) \equiv\left(\int_{0}^{k} d^{3} k^{\prime} X_{k^{\prime}}^{2}\right)^{1 / 2} .
$$

To estimate this integral, recall that the period of $P(x, k)$ is independent of $k$. To a good approximation we can take the Floquet exponent to be independent of $k$ as long as the mode $k$ is in the infrared instability band. Since we are interested in modes $k$ which are cosmological today, the assumption that $k$ lies in this band will be satisfied (recall that the infrared instability band runs from $k=0$ to $k=k_{c}$, where $k_{c}$ is a microscopic scale). For vacuum initial conditions the integral is dominated at the upper end $k^{\prime}=k$, and hence using 22 we obtain

$$
\chi_{\mathrm{eff}}(k) \sim \sqrt{\pi} H \exp (\mu x) P_{1}(x, k),
$$

where we have dropped the index on the Floquet exponent. The periodic function $P_{1}$ is [11] a series of sine and cosine functions with different coefficients and the leading term is $\sin (x)$ with unit coefficient Therefore, the leading term for $P_{1}$ gives us

$$
\chi_{\mathrm{eff}}(k) \sim \sqrt{\pi} H \exp (\mu x) \sin (x)
$$

for the effective background value of the preheat field.

\section{COVARIANT FORMALISM}

In this section we review the covariant formalism following the references $[38,50,52$ in which the formalism was developed (see also [53] for related work). The advantage of the covariant formalism is that it is based on variables which vanish identically for the background cosmology. Hence the fluctuations of these quantities are automatically gauge-invariant and can be used for a nonperturbative analysis [54.

In the following we first define the variables which are used in the covariant analysis. Then we determine the dynamical equations which they satisfy. We then make two simplifications. First, we take the long wavelength approximation which is well justified since we are interested in modes which are well outside the Hubble radius during reheating. Finally, we solve the resulting equations in the leading approximation.

\section{Case 1: A Perfect Fluid}

For instructive purposes we first review the covariant formalism in the context of perfect fluid matter. We consider space-time as a manifold with a preferred fluid flow direction which is characterized by a four-velocity $u^{a}$ which satisfies the normalization condition $u^{a} u_{a}=-1$.

The energy-momentum tensor for the perfect fluid is

$$
T_{b}^{a}=(\rho+P) u^{a} u_{b}+P g_{b}^{a}
$$

where $\rho$ and $P$ are energy density and pressure, respectively. The spatial projection tensor orthogonal to the fluid four-velocity is

$$
h_{a b} \equiv g_{a b}+u_{a} u_{b},
$$


and satisfies the relations

$$
\begin{aligned}
h_{b}^{a} h_{c}^{b} & =h_{c}^{a} \text { and } \\
h_{a}^{b} u_{b} & =0 .
\end{aligned}
$$

The expansion parameter of space is given by

$$
\Theta \equiv \nabla_{a} u^{a},
$$

where $\nabla_{a}$ is the covariant derivative. The acceleration, $u^{a}$, is defined through the projected covariant derivative along the four-velocity. To be more precise, let us define the time evolution of any quantity in the covariant formalism by the Lie derivative with respect to the flow direction in the manifold. For a one form $Y_{a}$ the Lie derivative is defined by

$$
\dot{Y}_{a} \equiv \mathcal{L}_{u} Y_{a}=u^{c} \nabla_{c} Y_{a}+Y_{c} \nabla_{a} u^{c} .
$$

For a scalar $f$ only the first term arises, i.e. $\dot{f}=u^{a} \nabla_{a} f$. Therefore the acceleration is defined by

$$
u^{a}=\mathcal{L}_{u} u^{a} .
$$

For each comoving observer, we can define the logarithm $\alpha$ of the local scale factor by integrating $\Theta$ along the fluid world lines:

$$
\int d \tau \Theta \equiv 3 \alpha
$$

Key to the covariant approach is to make use of variables which vanish on the unperturbed space-time. Following [38, 39, 55] one can define the "projected covariant derivative" operator

$$
D_{a} \equiv h_{a}^{b} \nabla_{b} .
$$

It is the projection onto the hypersurface perpendicular to the vector field tangent to the flow lines. Next, one can introduce the spatially projected covariant derivative (projected gradient) of the energy density

$$
D_{a} \rho \equiv h_{a}^{b} \nabla_{b} \rho=\partial_{a} \rho+u_{a} \dot{\rho},
$$

of the pressure

$$
D_{a} P \equiv h_{a}^{b} \nabla_{b} P=\partial_{a} P+u_{a} \dot{P}
$$

and of the expansion parameter

$$
D_{a} \Theta \equiv h_{a}^{b} \nabla_{b} \Theta=\partial_{a} \Theta+u_{a} \dot{\Theta} .
$$

As these quantities vanish in FRW space-time, they yield a fully geometrical and nonperturbative characterization of perturbations.

Knowing that, let us work out the generalized curvature and non-adiabatic pressure perturbation in a geometrical way. Starting point are the conservation equations for the energy-momentum tensor whose first component fields the continuity equation

$$
\dot{\rho}+\Theta(\rho+P)=0 .
$$

Using the projected gradient of this equation one can define the curvature covector as below [50]

$$
\zeta_{a} \equiv D_{a} \alpha-\frac{\dot{\alpha}}{\dot{\rho}} D_{a} \rho
$$

Then the time evolution of this quantity is

$$
\dot{\zeta}_{a}=\mathcal{L}_{u} \zeta_{a}=-\frac{\Theta}{3(\rho+P)}\left(D_{a} P-c_{s}^{2} D_{a} \rho\right)
$$

where $c_{s}^{2} \equiv \frac{\dot{P}}{\dot{\rho}}$ is the generalized speed of sound as defined in [50]. Comparing this equation with the familiar equation of motion in linear theory where the right-hand side of the equation is the non-adiabatic pressure perturbation, we define non-adiabatic pressure covector as

$$
\Gamma_{a} \equiv D_{a} P-\frac{\dot{P}}{\dot{\rho}} D_{a} \rho .
$$

Making use of the definitions in equations \begin{tabular}{l|l|l|l|l|l|l|}
31 & 32 & 34 & one
\end{tabular} can rewrite the curvature and non-adiabatic pressure covectors in terms of ordinary gradients

$$
\begin{gathered}
\zeta_{a}=\partial_{a} \alpha-\frac{\dot{\alpha}}{\dot{\rho}} \partial \rho \\
\Gamma_{a}=\partial P-\frac{\dot{P}}{\dot{\rho}} \partial \rho .
\end{gathered}
$$

For the case of a single scalar field one can show that non-adiabatic pressure covector is

$$
\Gamma_{a}=2 \frac{\dot{\phi}}{\dot{\rho}} V_{, \phi} D_{a} \rho
$$

which vanishes in the long wavelength approximation. Then considering $\dot{\rho}=-\Theta \dot{\phi}^{2}$ in equation (39), leads to the equation

$$
\dot{\zeta}_{a}=\frac{2}{3} \frac{V_{, \phi}}{\dot{\phi}^{3}} D_{a} \rho
$$

for the time evolution of the curvature covector for the case of a single scalar field. The right-hand side of this equation vanished in the long wavelength approximation. This yields the conclusion that in the case of a single perfect fluid the curvature fluctuation $\zeta$ is conserved on super-Hubble scales at arbitrary order in perturbation theory.

\section{Case 2: A System of Two Coupled Scalar Fields}

The extension to the case of two scalar fields was given in 50. The first step is to identify the adiabatic and the entropy components of the fluctuations in this two field system. To do this we use the formalism developed 
in [56] in which we are given two scalar fields $\phi$ and $\chi$ which both have non-vanishing backgrounds which are evolving in time. The adiabatic field $\sigma$ is tangent to the field trajectory, the entropy field $s$ is orthogonal to it. We can introduce the corresponding unit vectors in twodimensional field space via

$$
\begin{aligned}
e_{\sigma}^{I} & \equiv \frac{1}{\sqrt{\dot{\phi}^{2}+\dot{\chi}^{2}}}(\dot{\phi}, \dot{\chi}), \\
e_{s}^{I} & \equiv \frac{1}{\sqrt{\dot{\phi}^{2}+\dot{\chi}^{2}}}(-\dot{\chi}, \dot{\phi}),
\end{aligned}
$$

where $I$ is the field space index. The angle $\theta$ of the trajectory in field space is then given (in the small angle approximation) by

$$
\theta=\frac{\dot{\chi}}{\dot{\phi}}
$$

Using the above definitions, we can set up the adiabatic and entropy field covectors by taking the respective projective covariant derivatives of the basis fields $\phi$ and $\chi$ :

$$
\begin{aligned}
\sigma_{a} & \equiv e_{\sigma}^{I} \nabla_{a} \varphi_{I}=\cos \theta \nabla_{a} \phi+\sin \theta \nabla_{a} \chi \\
s_{a} & \equiv e_{s}^{I} \nabla_{a} \varphi_{I}=-\sin \theta \nabla_{a} \phi+\cos \theta \nabla_{a} \chi .
\end{aligned}
$$

Note that $s_{a}$ is orthogonal to $u^{a}$ and we have $u^{a} s_{a}=$ 0 , but this is not the case for adiabatic covector since $u^{a} \sigma_{a}=\dot{\sigma}$.

The geometrical variables which describe the field perturbations are obtained by taking the spatially projected version of the above equations

$$
\begin{aligned}
\sigma_{a}^{\perp} & \equiv e_{\sigma}^{I} D_{a} \varphi_{I}=\sigma_{a}+\dot{\sigma} u_{a} \\
s_{a}^{\perp} & \equiv e_{s}^{I} D_{a} \varphi_{I}=s_{a} .
\end{aligned}
$$

Note that these fluctuations are well defined nonperturbatively.

From the Klein-Gordon equations for the $\phi$ and $\chi$ fields, we can find the adiabatic and entropy components of the Klein-Gordon equations. Using these equations we can find the evolution equation for the adiabatic $\sigma_{a}$ and entropy $s_{a}$ covectors. The resulting equation for the adiabatic component is 50 .

$$
\begin{aligned}
\left(\ddot{\sigma}_{a}\right)^{\perp} & +\Theta(\dot{\sigma})_{a}^{\perp}+\dot{\sigma} D_{a} \Theta+\left(V_{, \sigma \sigma}+\dot{\theta} \frac{V_{, s}}{\dot{\sigma}}\right) \sigma_{a}^{\perp}-D_{a}\left(\nabla^{c} \sigma_{c}^{\perp}\right) \\
& \left.=\left(\dot{\theta}-\frac{V_{, s}}{\dot{\sigma}}\right) s_{a}\right)^{\cdot}+\left(\ddot{\theta}-V_{, s \sigma}+\Theta \dot{\theta}\right) s_{a}-D_{a} Y_{(s)},(52)
\end{aligned}
$$

where

$$
Y_{(s)}=\frac{1}{\dot{\sigma}}\left(\dot{s}_{a}+\dot{\theta} \sigma_{a}^{\perp}\right) s^{a} .
$$

The equation for the entropy component is [50]

$$
\ddot{s}_{a}+\left(\Theta-\frac{1}{\dot{\sigma}}\left(\nabla^{c} \sigma_{c}^{\perp}-Y_{(s)}\right)\right) \dot{s}_{a}+\left(V_{, s s}+\dot{\theta}^{2}-2 \dot{\theta} \frac{V_{, s}}{\dot{\sigma}}\right) s_{a}
$$

$$
\begin{aligned}
& -D_{a}\left(\nabla_{c} s^{c}\right) \\
= & \frac{\dot{\theta}}{\dot{\sigma}}\left(D_{a} \Pi-\frac{\Pi}{\dot{\sigma}} \sigma_{a}^{\perp}-2 \epsilon_{a}\right)-\frac{1}{\dot{\sigma}}\left(D_{c} s^{c}+Y_{(\sigma)}\right) \dot{\sigma_{a}^{\perp}} \\
& +D_{a} Y_{(\sigma)}
\end{aligned}
$$

where $\epsilon_{a}$ is the covector associated with the comoving energy density perturbation and

$$
Y_{(\sigma)} \equiv \frac{1}{\dot{\sigma}}\left(\dot{s}_{a}+\dot{\theta} \sigma_{a}^{\perp}\right) \sigma^{\perp, a} .
$$

The first approximation we make is to linearize these equations (the expansion parameter is the amplitude of the fluctuations, which in our case is proportional to $\hbar$.) This yields greatly simplified equations

$$
\begin{aligned}
\left(\ddot{\sigma}_{a}\right)^{\perp} & +3 H(\dot{\sigma})_{a}^{\perp}+\dot{\sigma} D_{a} \Theta+\left(V_{, \sigma \sigma}-\dot{\theta}^{2}\right) \sigma_{a}^{\perp}-D_{a}\left(D^{c} \sigma_{c}^{\perp}\right) \\
& \simeq 2\left(\dot{\theta} s_{a}\right)^{\cdot}-2 \frac{V_{, s}}{\dot{\sigma}} \dot{\theta} s_{a}
\end{aligned}
$$

and

$$
\begin{aligned}
\ddot{s}_{a} & +3 H \dot{s}_{a}+\left(V_{, s s}+3 \dot{\theta}^{2}\right) s_{a}-D_{a}\left(D_{c} s^{c}\right) \\
& \simeq-2 \frac{\dot{\theta}}{\dot{\sigma}} \epsilon_{a} .
\end{aligned}
$$

The final approximation we make is to focus on long wavelengths, i.e. we work in the leading order gradient expansion in which also the comoving energy density fluctuation vanishes. This yields our final equations

$$
\begin{aligned}
\left(\ddot{\sigma}_{a}\right)^{\perp} & +3 H(\dot{\sigma})_{a}^{\perp}+\dot{\sigma} D_{a} \Theta+\left(V_{, \sigma \sigma}-\dot{\theta}^{2}\right) \sigma_{a}^{\perp} \\
& \simeq 2\left(\dot{\theta} s_{a}\right)^{\cdot}-2 \dot{\theta} \frac{V_{, \sigma}}{\dot{\sigma}} s_{a}
\end{aligned}
$$

and

$$
\ddot{s}_{a}+3 H \dot{s}_{a}+\left(V_{, s s}+3 \dot{\theta}^{2}\right) s_{a} \simeq 0 .
$$

As is well known in the linear theory of cosmological perturbations (see e.g. [57] for an overview and [58] for an introduction), the entropy fluctuations are not affected by the amplitude of the adiabatic perturbation. On the other hand, entropy fluctuations induce a growing adiabatic mode.

\section{APPLICATION OF THE COVARIANT FORMALISM TO MASSLESS PREHEATING}

Our goal in this section is to show that in massless preheating entropy fluctuations are indeed parametrically amplified, and that this in turn leads to an exponentially growing contribution to curvature fluctuations. As we shall see, the effect on the curvature fluctuations is quadratic in the amplitude of the quantum fluctuations. The entropy fluctuations themselves have an exponentially growing term which is linear in the fluctuation amplitude. However, the coupling between the entropy and 
the adiabatic mode is suppressed by an additional power which comes from the fact that the background of the entropy field vanishes at zero'th order.

For the case of massless preheating the equation for long wave linearized entropy fluctuations $(59)$ becomes

$$
\begin{aligned}
\ddot{s}_{a} & +3 H \dot{s}_{a}+\left[\left(3 \lambda \phi^{2}+g^{2} \chi^{2}\right) \sin ^{2} \theta-2 g^{2} \phi \chi \sin 2 \theta\right. \\
& \left.+g^{2} \phi^{2} \cos ^{2} \theta+3 \dot{\theta}^{2}\right] s_{a} \simeq 0 .
\end{aligned}
$$

To analyze this equation of motion we make a couple of approximations and use the following setup:

- As mentioned before, at the beginning of preheating the overall homogeneous value of the $\chi$ field is zero (the effective $\chi$ field on a scale $k$ will be nonvanishing but of linear order in the amplitude of the fluctuations).

- We use the relation for $\theta$ in the small angle approximation introduced in the previous subsection:

$$
\theta \equiv \frac{\dot{\chi}}{\dot{\phi}}
$$

for the instantaneous angle between the background trajectory and the $\phi$ field direction in field space. Therefore at the beginning of preheating the angle is of linear order in the fluctuations.

- We will use the result from [56] for $\dot{\theta}$ in the large scale limit

$$
\dot{\theta}=-\frac{V_{, s}}{\dot{\sigma}},
$$

where $V, s$ is the derivative of the potential with respect to the entropy component. We use another result which is shown in [56]

$$
V_{, s}=-V_{, \phi} \sin \theta+V_{, \chi} \cos \theta .
$$

At the beginning of preheating we have $V_{, \chi} \simeq \chi$ which like $\chi$ is of first order. Thus, $\dot{\theta}$ is of first order and we can drop the last term in equation (60) as it is of second order.

- The perturbative expression for the expansion parameter is

$\Theta=3 H+\epsilon F+\epsilon^{2} G+$ higher order terms .

We will only need to consider the zero'th order term since effects of the other terms would be of higher order in perturbation theory.

Making use of the above points, the equation (59) at leading order becomes

$$
\ddot{s}_{a}+3 H \dot{s}_{a}+g^{2} \phi^{2} s_{a}=0 .
$$

If we do a conformal transformation

$$
\begin{aligned}
a s_{a} & \equiv S_{a} \\
a \phi & \equiv \varphi
\end{aligned}
$$

and work with conformal time instead of physical time we get

$$
S_{a}^{\prime \prime}+g^{2} \varphi^{2} S_{a}=0
$$

which has the same structure as the equations 14 , 15 for the $\chi$ background field. From the discussion of the solutions of this equation in the early section of this article it thus follows that

$$
S_{a}=B_{1} u_{1 a}+B_{2} u_{2 a},
$$

where $u_{1 a}$ is exponentially growing and $u_{2 a}$ is exponentially damped. Considering only the growing mode and remembering the same form for $u_{1 a}$ as we used in equation 22, we find

$$
S_{a}=H k^{-3 / 2} \exp (\mu x) P_{1 a}(x, k),
$$

where $P_{1 a}$ indicate periodic functions of $x$ with unit amplitude, and we hence conclude that the entropy component is exponentially growing due to parametric resonance at the beginning of preheating. This is one of the main results of our paper.

We are interested in the process of conversion of the entropy fluctuation into a curvature fluctuation. This process happens continuously througout the preheating phase. In the rest of this section we will study the evolution of curvature covector due to this conversion process.

As is well known, entropy fluctuations can seed a growing curvature fluctuation mode on super-Hubble scales. In the linear approximation which we use (and working under the small angle $\theta$ assumption) the induced curvature fluctuation $\zeta_{k}^{e n t}$ is given by

$$
\zeta_{k}^{e n t} \simeq \frac{H}{\dot{\varphi}^{2}} \dot{\chi} S_{k},
$$

where $S_{k}$ is the Fourier space entropy fluctuation determined above in 697. Making use of 69), inserting the result for $\chi$ given in (25), and taking care of the change in the temporal variable from $x$ to $\eta$ we find

$$
\zeta_{k}^{e n t} \simeq \sqrt{\pi \lambda} \frac{H^{3} \varphi}{\dot{\varphi}^{2}} e^{2 \mu x} k^{-3 / 2} P(x, k)
$$

where $P$ is a periodic function of unit amplitude. This clearly shows the exponential growth of the induced curvature fluctuations. A derivation of this result from first principles making use of the covariant formalism is given in the Appendix. Note that even though $\mu_{k}<1$, the time scale of the exponential instability is (while long compared to the oscillation time) short compared to the 
Hubble expansion time, the time scale relevant to the conversion of entropy fluctuations to adiabatic ones.

We can evaluate the power spectrum for the curvature perturbation given by the entropy mode from at horizon crossing:

$$
P_{k}^{e n t}=\frac{k^{3}}{2 \pi^{2}}\left|\zeta_{k}^{e n t}\right|^{2} \sim \lambda \frac{H^{6}}{\dot{\varphi}^{4}} \varphi^{2} e^{4 \mu x} .
$$

Hence, we conclude that the entropy perturbations give a scale invariant contribution to the power spectrum of the curvature perturbation from inflation, but with an amplitude which is exponentially increasing.

We have derived our result in a simple two field inflation model, the conclusions will carry over to other multi-field models. Our work suggests that in any inflationary model in which the inflaton satisfies the massless preheating condition, then if low mass entropy fields are present which couple to the inflaton, then parametric resonance of the entropy perturbation indeed happens. Due to the conversion process of entropy perturbation into adiabatic perturbation (as studied in the context of coupled scalar fields in [56]), parametric resonance of entropy perturbation may lead to a rapidly growing adiabatic mode which could have a large impact on the spectrum of curvature perturbation we observe today. The spectrum will remain approximately scale-invariant, but there is the danger that the exponential growth will cause the fluctuations to become non-linear (which would rule out the model). To see whether this is a serious concern, we must however first consider back-reaction issues. Backreaction might cut off the instability before the induced curvature fluctuations become too large. However, below we find that at least the back-reaction effects which we consider are not strong enough to shut off the resonance in time for the induced curvature fluctuations to remain small enough.

\section{Back-reaction}

In the previous sections we considered the parametric resonance of entropy perturbations during preheating neglecting any back-reaction effects. However, the exponential instability of the entropy field leads to an exponential creation of $\chi$ particles that are expected to back react in the background. The study of back-reaction is important, since the cumulative effect of the creation of particle eventually becomes important affecting the resonance and even terminating preheating, as already noticed in [8, 28, 47] (see also [59] for earlier numerical work).

We will consider two back-reaction effects that can affect preheating in the $g^{2} / \lambda=2$ case. Other back-reaction effects and parameters choices were studied in 28, 47]. The first effect is the back reaction of the parametrically amplified $\chi$ on the evolution of the inflaton background.
If the force induced by $\chi$ is larger than the force present in the absence of $\chi$, then the condition for massless preheating will no longer be satisfied and the broad parametric resonance will terminate. This will happen when

$$
g^{2}\left\langle\chi^{2}\right\rangle \sim \lambda \phi^{2}
$$

Using $\left\langle\chi^{2}\right\rangle=\chi_{\text {eff }}^{2}$ from 25 and setting $\phi=\phi_{\text {end }}$, this condition implies that

$$
e^{2 \mu \Delta x} \simeq \lambda^{-1}
$$

This gives us the time interval before this back-reaction effect becomes important. We can use this result to evaluate the power spectrum of the curvature perturbations induced by entropy modes at the time that the resonance shuts off by this back-reaaction effect. Using $(72)$ and (74), the power spectrum at this time is given by:

$$
P_{k}^{e n t} \sim \lambda^{-1} \frac{H^{6}}{\dot{\varphi}^{4}} \varphi^{2} .
$$

Since at the end of inflation $\dot{\varphi}^{2}=V$ and $\varphi=\varphi_{\text {end }}$, we can estimate the power spectrum for the curvature perturbations from the super-Hubble amplified entropy perturbations as:

$$
P_{k}^{e n t} \sim \frac{1}{5}
$$

This is considerably larger than the observed values, exceeding by many orders of magnitude the COBE normalization measurement 60 . We thus conclude that the parametric amplification of entropy perturbations can lead to a serious problem for models like the one we consider, unless other effects are found which shut off the resonance earlier.

We can also consider the back-reaction of the produced $\chi$ particles on the Friedmann equation. We find that demanding that the induced $\chi$ terms remain sub-dominant leads to precisely the condition (73).

The second effect considered in this paper is the influence of the produced $\delta \phi$ particles on the $\delta \chi$ resonance. If the creation of $\phi$ particles is large enough, increasing significantly the effective mass of the $\delta \chi$ field, this could damp or even stop the resonance of the $\chi$ field. Thus we need to know if

$$
V_{, \chi}<g^{2} \chi\left\langle\delta \phi^{2}\right\rangle \Rightarrow \phi^{2}<\left\langle\delta \phi^{2}\right\rangle,
$$

at some point during preheating, altering the effective time dependent mass and consequently the $\chi$ resonance. However, we can see from the equation for the eigenmodes $\phi_{k}(t)$ during preheating:

$$
\delta \ddot{\phi}_{k}+3 H \delta \dot{\phi}_{k}+\left(\frac{k^{2}}{a^{2}}+3 \lambda \delta \phi^{2}\right) \delta \phi_{k}=0,
$$

that for $\delta \phi_{k}$ the resonance is always narrow, since it is equivalent to the case of parametric resonance with 
$g^{2} / \lambda=3$. This leads to a very small characteristic exponent $\mu$ [4] and a very inefficient creation of $\phi$ particles. This effect will always be less important than the parametric resonance for the $\chi$ field, that is very broad and has a large characteristic exponent. Thus, the second back-reaction effect studied in this paragraph does not have the potential of shutting off the resonant amplification of entropy fluctuations early, and does not change the conclusion from 76 that the curvature perturbations are too large when the resonance is finally shut off.

We have to stress, though, that the full theory of back reaction and reescatering during preheating is not fully developed. However, this result represents an advance with respect to previous investigations since the covariant formalism allows for a full non-linear analysis including metric fluctuations (although we here considered only the linear limit).

\section{CONCLUSIONS}

We have considered the preheating of entropy fluctuations in a two field model in which an inflaton field with vanishing bare mass is coupled to a massless entropy field. In the absence of metric fluctuations, it is known that in this model there is efficient preheating ("massless preheating"). We find, using a covariant formulation of the theory of cosmological fluctuations which in principle can be extended to a full nonlinear analysis, that the entropy fluctuations experience a period of broad parametric resonance. At quadratic order in the amplitude of fluctuations, the entropy modes seed a growing curvature fluctuation. Hence, we find a curvature fluctuation mode which is growing exponentially during the preheating phase. In agreement with previous studies [28] we find that back-reaction effects are too weak to shut off the resonance before the power spectrum of the induced curvature fluctuations has reached an amplitude close to 1, i.e. many orders of magnitude larger than the observational value. Hence we see that models of the type we consider here are phenomenologically ruled out, unless there are back-reaction effects not considered here which manage to truncate the resonance earlier than the ones we have studied.

Our results confirm those of earlier studies [24, 25] which were reached using different methods. The advantage of the formalism used here is that it is in principle extendible to the non-linear regime, although here we use a linear truncation of the method. Hence, our paper resolves a controversy about the validity of previous works.

A significant limitation of our results is that they only apply to models with a massless inflaton. Such models may be motivated by conformal symmetry arguments. We do not regard the restriction to massless entropy fields to be a significant limitation, the reason being that the conclusions go through as long as the $\chi$ mass is small compared to $H$, and there are lots of fields with masses smaller than the value of $H$ during inflation.

\section{ACKNOWLEDGEMENTS}

We thank A. Abolhasani, M. Oltean, L. Perreault Levasseur, M. Namjoo, M. Sasaki, T. Suyama and Y. Wang for useful discussions. This work was supported in part by a NSERC Discovery grant and by funds from the Canada Research Chair program (RB).

\section{APPENDIX}

In this appendix we derive the growth of induced curvature fluctuations from first principles, making use of the covariant formalism.

In the long wavelength limit, the growth of $\zeta$ is given by 38,50 .

$$
\dot{\zeta}_{a} \equiv \mathcal{L}_{u} \zeta_{a}=\frac{2}{3} \frac{\Theta}{\dot{\sigma}^{2}} V_{, s} S_{a}
$$

Note that the induced growing mode of $\zeta$ is quadratic in the magnitude of fluctuations.

The equation 79 can be understood in the following way: For the Lie derivative of curvature covector we have

$$
\mathcal{L}_{u} \zeta_{a}=u^{c} \nabla_{c} \zeta_{a}+\zeta_{c} \nabla_{a} u^{c}
$$

Considering $u^{c}=\{1 / a, 0,0,0\}$ we get

$$
\mathcal{L}_{u} \zeta_{a}=\frac{1}{a} \partial_{t} \zeta_{a}
$$

Then one can find the relation between the curvature covector and the conventional coordinate based curvature perturbation as follows [52] (up to first order)

$$
\zeta_{i}=\partial_{i} \zeta
$$

Note that the quantity $\zeta$ we introduced here is the same as the conventional curvature perturbation in the large scale limit which we are interested in (see the discussion in [52]). The same relation holds for $S_{a}$, and considering $\partial_{i} \rightarrow i k$ in Fourier space leads to

$$
\frac{1}{a} \partial_{t} \zeta=\frac{\sqrt{2} H}{\dot{\sigma}^{2}} V_{, s} H k^{-3 / 2} \exp (\mu x) P_{1}(x) .
$$

Up to first order in perturbation theory

$$
\dot{\sigma}^{2} \simeq \dot{\phi}^{2}
$$

and

$$
V_{, s}=-\lambda \phi^{3} \theta+g^{2} \phi^{2} \chi
$$


Using dimensionless conformal time and again applying the conformal transformation $\varphi=a \phi$ and the same for the preheat field $\chi$, we obtain for the angle $\theta$

$$
\theta=\frac{\frac{\chi^{\prime}}{a}-\frac{a^{\prime}}{a^{2}} \chi}{\frac{\varphi^{\prime}}{a}-\frac{a^{\prime}}{a^{2}} \varphi}
$$

Therefore using equation 79 gives

$$
\zeta^{\prime}=\frac{2 \sqrt{\pi}}{x} k^{-3 / 2} \cos ^{2}(x) e^{2 \mu x} F(x),
$$

where

$$
F(x) \equiv \lambda \frac{\mu x \sin ^{2}(x) \cos (x)+x \cos ^{2}(x) \sin (x)-\sin ^{2}(x) \cos (x)}{(x \sin (x)+\cos (x))^{3}}+g^{2} \frac{\sin ^{2}(x)}{(x \sin (x)+\cos (x))^{2}},
$$

which clearly shows that

$$
\zeta \propto \exp (2 \mu x)
$$

Hence, we conclude that parametric resonance of entropy perturbations induces an exponentially growing curvature mode.

Thus, we see that taking into account the squeezing of the modes and the solution 22 , the curvature perturbation induced by the entropy modes after inflation, using (83), is given by:

$$
\zeta_{k}^{e n t} \sim \sqrt{2 \pi \lambda} \frac{H^{3}}{\dot{\varphi}^{2}} \varphi k^{-3 / 2} e^{2 \mu x}
$$

where we made the approximation of $V_{, s} \simeq \lambda \varphi^{2} \chi_{\text {eff } f \text { since }}$ $g^{2} / \lambda=2$, the same result obtained in the main part of the text using the approximate treatment.

* Electronic address: bazrafshan, rhb, yifucai, elisafenu@physics.mcgill.ca

[1] L. F. Abbott, E. Farhi and M. B. Wise, "Particle Production In The New Inflationary Cosmology," Phys. Lett. B 117, 29 (1982).

[2] A. D. Dolgov and A. D. Linde, "Baryon Asymmetry In Inflationary Universe," Phys. Lett. B 116, 329 (1982).

[3] A. J. Albrecht, P. J. Steinhardt, M. S. Turner and F. Wilczek, "Reheating An Inflationary Universe," Phys. Rev. Lett. 48, 1437 (1982).

[4] J. H. Traschen and R. H. Brandenberger, "Particle Production During Out-Of-Equilibrium Phase Transitions," Phys. Rev. D 42, 2491 (1990).

[5] A. D. Dolgov and D. P. Kirilova, "On Particle Creation By A Time Dependent Scalar Field," Sov. J. Nucl. Phys. 51, 172 (1990) [Yad. Fiz. 51, 273 (1990)].

[6] L. Kofman, A. D. Linde and A. A. Starobinsky, "Reheating after inflation," Phys. Rev. Lett. 73, 3195 (1994) arXiv:hep-th/9405187.

[7] Y. Shtanov, J. H. Traschen and R. H. Brandenberger, "Universe reheating after inflation," Phys. Rev. D 51, 5438 (1995) arXiv:hep-ph/9407247.

[8] L. Kofman, A. D. Linde and A. A. Starobinsky, "Towards the theory of reheating after inflation," Phys. Rev. D 56, 3258 (1997) arXiv:hep-ph/9704452.
[9] R. Allahverdi, R. Brandenberger, F. Y. Cyr-Racine and A. Mazumdar, "Reheating in Inflationary Cosmology: Theory and Applications," arXiv:1001.2600 [hep-th].

[10] M. A. Amin, M. P. Hertzberg, D. I. Kaiser and J. Karouby, "Nonperturbative Dynamics Of Reheating After Inflation: A Review," Int. J. Mod. Phys. D 24, no. 01, 1530003 (2014) arXiv:1410.3808 [hep-ph]].

[11] N. McLachlan, "Theory and Applications of Mathieu Functions" (Oxford Univ. Press, Clarendon, 1947).

[12] Y. Nambu and A. Taruya, "Evolution of cosmological perturbation in reheating phase of the universe," Prog. Theor. Phys. 97, 83 (1997) gr-qc/9609029.

[13] B. A. Bassett, D. I. Kaiser and R. Maartens, "General relativistic preheating after inflation," Phys. Lett. B 455, 84 (1999) arXiv:hep-ph/9808404;

B. A. Bassett, F. Tamburini, D. I. Kaiser and R. Maartens, "Metric preheating and limitations of linearized gravity. II," Nucl. Phys. B 561, 188 (1999) arXiv:hep-ph/9901319.

[14] F. Finelli and R. H. Brandenberger, "Parametric amplification of gravitational fluctuations during reheating," Phys. Rev. Lett. 82, 1362 (1999) arXiv:hep$\mathrm{ph} / 9809490$.

[15] K. Jedamzik and G. Sigl, "On metric preheating," Phys. Rev. D 61, 023519 (2000) arXiv:hep-ph/9906287;

P. Ivanov, "On generation of metric perturbations during preheating," Phys. Rev. D 61, 023505 (2000) arXiv:astro-ph/9906415;

A. R. Liddle, D. H. Lyth, K. A. Malik and D. Wands, "Super-horizon perturbations and preheating," Phys. Rev. D 61, 103509 (2000) arXiv:hep-ph/9912473;

W. B. Lin, X. H. Meng and X. M. Zhang, "Adiabatic gravitational perturbation during reheating," Phys. Rev. D 61, 121301 (2000) arXiv:hep-ph/9912510.

[16] H. Kodama and T. Hamazaki, "Evolution of cosmological perturbations in a stage dominated by an oscillatory scalar field," Prog. Theor. Phys. 96, 949 (1996) grqc/9608022.

[17] Y. Nambu and A. Taruya, "Solutions of gauge invariant cosmological perturbations in long wavelength limit," Class. Quant. Grav. 15, 2761 (1998) gr-qc/9801021.

[18] N. Afshordi and R. H. Brandenberger, "Super Hubble nonlinear perturbations during inflation," Phys. Rev. D 63, 123505 (2001) gr-qc/0011075.

[19] D. Langlois and F. Vernizzi, "Evolution of non-linear cosmological perturbations," Phys. Rev. Lett. 95, 091303 (2005) astro-ph/0503416; 
D. Langlois and F. Vernizzi, "Conserved non-linear quantities in cosmology," Phys. Rev. D 72, 103501 (2005) astro-ph/0509078.

[20] J. M. Bardeen, "Gauge Invariant Cosmological Perturbations," Phys. Rev. D 22, 1882 (1980).

[21] J. M. Bardeen, P. J. Steinhardt and M. S. Turner, "Spontaneous Creation of Almost Scale - Free Density Perturbations in an Inflationary Universe," Phys. Rev. D 28, 679 (1983).

[22] R. H. Brandenberger and R. Kahn, "Cosmological Perturbations In Inflationary Universe Models," Phys. Rev. D 29, 2172 (1984).

[23] A. Taruya and Y. Nambu, "Cosmological perturbation with two scalar fields in reheating after inflation," Phys. Lett. B 428, 37 (1998) arXiv:gr-qc/9709035.

[24] B. A. Bassett and F. Viniegra, "Massless metric preheating," Phys. Rev. D 62, 043507 (2000) arXiv:hep$\mathrm{ph} / 9909353$.

[25] F. Finelli and R. H. Brandenberger, "Parametric amplification of metric fluctuations during reheating in two field models," Phys. Rev. D 62, 083502 (2000) arXiv:hep$\mathrm{ph} / 0003172$.

[26] R. Easther and M. Parry, "Gravity, parametric resonance and chaotic inflation," Phys. Rev. D 62, 103503 (2000) hep-ph/9910441.

[27] T. Hamazaki and H. Kodama, "Evolution of cosmological perturbations during reheating," Prog. Theor. Phys. 96, 1123 (1996) gr-qc/9609036.

[28] J. P. Zibin, R. H. Brandenberger and D. Scott, "Back reaction and the parametric resonance of cosmological fluctuations," Phys. Rev. D 63, 043511 (2001) hep$\mathrm{ph} / 0007219$.

[29] L. Perreault Levasseur, G. Laporte and R. Brandenberger, "Analytical Study of Mode Coupling in Hybrid Inflation," Phys. Rev. D 82, 123524 (2010) arXiv:1004.1425 [hep-th]];

L. Perreault Levasseur, "Lagrangian formulation of stochastic inflation: Langevin equations, one-loop corrections and a proposed recursive approach," Phys. Rev. D 88, no. 8, 083537 (2013) arXiv:1304.6408 [hep-th]].

[30] A. B. Henriques and R. G. Moorhouse, "Cosmic microwave background and parametric resonance in reheating," Phys. Rev. D 65, 103524 (2002) hep-ph/0109218.

[31] S. Tsujikawa and B. A. Bassett, "When can preheating affect the CMB?," Phys. Lett. B 536, 9 (2002) astro$\mathrm{ph} / 0204031$.

[32] F. Di Marco, F. Finelli and R. Brandenberger, "Adiabatic and isocurvature perturbations for multifield generalized Einstein models," Phys. Rev. D 67, 063512 (2003) astro-ph/0211276.

[33] F. -Y. Cyr-Racine and R. H. Brandenberger, "Study of the Growth of Entropy Modes in MSSM Flat Directions Decay: Constraints on the Parameter Space," JCAP 0902, 022 (2009) arXiv:0811.4457 [hep-ph]];

R. H. Brandenberger, A. R. Frey and L. C. Lorenz, "Entropy fluctuations in brane inflation models," Int. J. Mod. Phys. A 24, 4327 (2009) arXiv:0712.2178 [hep-th]]; M. Bastero-Gil, V. Di Clemente and S. F. King, "Preheating curvature perturbations with a coupled curvaton," Phys. Rev. D 70, 023501 (2004) hep-ph/0311237.

[34] T. Tanaka and B. Bassett, "Application of the separate universe approach to preheating," astro-ph/0302544.

[35] T. Suyama and S. Yokoyama, "Generating the primordial curvature perturbations in preheating," Class. Quant.
Grav. 24, 1615 (2007) astro-ph/0606228.

[36] T. Suyama and S. Yokoyama, "Statistics of general functions of a Gaussian field-application to nonGaussianity from preheating," JCAP 1306, 018 (2013) arXiv:1303.1254 [astro-ph.CO]].

[37] J. R. Bond, A. V. Frolov, Z. Huang and L. Kofman, "Non-Gaussian Spikes from Chaotic Billiards in Inflation Preheating," Phys. Rev. Lett. 103, 071301 (2009) arXiv:0903.3407 [astro-ph.CO]].

[38] D. Langlois and F. Vernizzi, "Evolution of non-linear cosmological perturbations," Phys. Rev. Lett. 95, 091303 (2005) astro-ph/0503416;

D. Langlois and F. Vernizzi, "Conserved non-linear quantities in cosmology," Phys. Rev. D 72, 103501 (2005) astro-ph/0509078.

[39] G. F. R. Ellis and M. Bruni, "Covariant and Gauge Invariant Approach to Cosmological Density Fluctuations," Phys. Rev. D 40, 1804 (1989).

[40] Z. Huang, "The Art of Lattice and Gravity Waves from Preheating," Phys. Rev. D 83, 123509 (2011) arXiv:1102.0227 [astro-ph.CO]];

Z. Huang, "A Cosmology Forecast Toolkit - CosmoLib," JCAP 1206, 012 (2012) arXiv:1201.5961 [astro-ph.CO]].

[41] P. Adshead, R. Easther, J. Pritchard and A. Loeb, "Inflation and the Scale Dependent Spectral Index: Prospects and Strategies," JCAP 1102, 021 (2011) arXiv:1007.3748 [astro-ph.CO]].

[42] A. V. Frolov, "DEFROST: A New Code for Simulating Preheating after Inflation," JCAP 0811, 009 (2008) arXiv:0809.4904 [hep-ph]].

[43] G. Felder and I. Tkachev, Comp. Phys. Comm.178 929 (2008);

J. F. Dufaux, A. Bergman, G. N. Felder, L. Kofman and J. P. Uzan, "Theory and Numerics of Gravitational Waves from Preheating after Inflation," Phys. Rev. D 76, 123517 (2007) arXiv:0707.0875 [astro-ph]];

J. F. Dufaux, G. Felder, L. Kofman and O. Navros, "Gravity Waves from Tachyonic Preheating after Hybrid Inflation," JCAP 0903, 001 (2009) arXiv:0812.2917 [astro-ph]].

[44] A. Chambers and A. Rajantie, "Lattice calculation of non-Gaussianity from preheating," Phys. Rev. Lett. 100, 041302 (2008) [Erratum-ibid. 101, 149903 (2008)] arXiv:0710.4133 [astro-ph]].

[45] G. N. Felder, J. Garcia-Bellido, P. B. Greene, L. Kofman, A. D. Linde and I. Tkachev, "Dynamics of symmetry breaking and tachyonic preheating," Phys. Rev. Lett. 87, 011601 (2001) hep-ph/0012142.

[46] S. Y. Khlebnikov and I. I. Tkachev, "Classical decay of inflaton," Phys. Rev. Lett. 77, 219 (1996) hepph/9603378;

S. Y. Khlebnikov and I. I. Tkachev, "Relic gravitational waves produced after preheating," Phys. Rev. D 56, 653 (1997) hep-ph/9701423.

[47] P. B. Greene, L. Kofman, A. D. Linde and A. A. Starobinsky, "Structure of resonance in preheating after inflation," Phys. Rev. D 56, 6175 (1997) hep-ph/9705347.

[48] P. Martineau, "On the decoherence of primordial fluctuations during inflation," Class. Quant. Grav. 24, 5817 (2007) astro-ph/0601134.

[49] C. Kiefer, D. Polarski and A. A. Starobinsky, "Quantum to classical transition for fluctuations in the early universe," Int. J. Mod. Phys. D 7, 455 (1998) grqc/9802003; 
C. Kiefer, I. Lohmar, D. Polarski and A. A. Starobinsky, "Pointer states for primordial fluctuations in inflationary cosmology," Class. Quant. Grav. 24, 1699 (2007) astro$\mathrm{ph} / 0610700$.

[50] D. Langlois and F. Vernizzi, "Nonlinear perturbations of cosmological scalar fields," JCAP 0702, 017 (2007) astro-ph/0610064.

[51] D. Langlois, F. Vernizzi and D. Wands, "Non-linear isocurvature perturbations and non-Gaussianities," JCAP 0812, 004 (2008) arXiv:0809.4646 [astro-ph]].

[52] D. Langlois and F. Vernizzi, "A geometrical approach to nonlinear perturbations in relativistic cosmology," Class. Quant. Grav. 27, 124007 (2010) arXiv:1003.3270 [astroph.CO]].

[53] G. I. Rigopoulos and E. P. S. Shellard, "The separate universe approach and the evolution of nonlinear superhorizon cosmological perturbations," Phys. Rev. D 68, 123518 (2003) astro-ph/0306620;

G. I. Rigopoulos and E. P. S. Shellard, "Non-linear inflationary perturbations," JCAP 0510, 006 (2005) astroph/0405185;

G. I. Rigopoulos, E. P. S. Shellard and B. J. W. van Tent, "Non-linear perturbations in multiple-field inflation," Phys. Rev. D 73, 083521 (2006) astro-ph/0504508.

[54] J. M. Stewart and M. Walker, "Perturbations of spacetimes in general relativity," Proc. Roy. Soc. Lond. A 341, 49 (1974).

[55] M. Bruni, G. F. R. Ellis and P. K. S. Dunsby, "Gauge invariant perturbations in a scalar field dominated universe," Class. Quant. Grav. 9, 921 (1992).

[56] C. Gordon, D. Wands, B. A. Bassett and R. Maartens, "Adiabatic and entropy perturbations from inflation," Phys. Rev. D 63, 023506 (2001) astro-ph/0009131.

[57] V. F. Mukhanov, H. A. Feldman and R. H. Branden- berger, "Theory of cosmological perturbations. Part 1. Classical perturbations. Part 2. Quantum theory of perturbations. Part 3. Extensions," Phys. Rept. 215, 203 (1992).

[58] R. H. Brandenberger, "Lectures on the theory of cosmological perturbations," Lect. Notes Phys. 646, 127 (2004) arXiv:hep-th/0306071.

[59] S. Y. Khlebnikov and I. I. Tkachev, "Classical decay of inflaton," Phys. Rev. Lett. 77, 219 (1996) hep$\mathrm{ph} / 9603378$;

S. Y. Khlebnikov and I. I. Tkachev, "The Universe after inflation: The Wide resonance case," Phys. Lett. B 390 , 80 (1997) hep-ph/9608458;

S. Y. Khlebnikov and I. I. Tkachev, "Resonant decay of Bose condensates," Phys. Rev. Lett. 79, 1607 (1997) hep-ph/9610477.

[60] E. F. Bunn, A. R. Liddle and M. J. White, 1, "Four year COBE normalization of inflationary cosmologies," Phys. Rev. D 54, 5917 (1996) astro-ph/9607038.

[61] In the case of "massive preheating" (the potential of the inflaton being dominated by the mass term) there is no preheating of the entropy mode at linear order in cosmological perturbation theory, as shown in 27.

[62] This is a key point in our analysis. At strictly linear order in perturbation theory the background value of $\chi$ would vanish. However, in a particular patch of length $k^{-1}$ the average value of $\chi$ will not vanish because of the presence of fluctuations on scales which are in the infrared with respect to $k$. They will produce a non-vanishing effective background. Our procedure corresponds to integrating out infrared modes which are unobservable from the point of view of the patch of interest. 